\title{
Der Arzt als Unternehmer und Manager
}

\section{Hans Stalder}

Unternehmensberater mit Fachgebiet Management

Interessenbindungen: Der Autor ist Anbieter eines Onlinelehrgangs für Praxismanagement.
Korrespondenz:

Hans Stalder

Hans Stalder + Partner GmbH

Schlossstrasse 44

CH-3672 Oberdiessbach

Tel. 0317712300

h.stalder@profkomm.ch
Aus der Sicht der Managementlehre ist jede Arztpraxis den rund 260000 Kleinbetrieben, die es in der Schweiz gibt, gleichzusetzen. Wie in jedem Betrieb gibt es in einer Arztpraxis die Rollen Fachkraft, Manager und Unternehmer. Während in grossen Betrieben die Rollen auf mehrere Personen verteilt sind, werden diese Aufgaben in KMU meistens von einer einzigen Person wahrgenommen. So auch in der Arztpraxis. Durch den hohen zeitlichen Einsatz im Umgang mit Patienten besteht dabei die Gefahr, dass der Arzt hauptsächlich in der Praxis und zu wenig an der Praxis arbeitet.

\section{Gibt es Rollenkonflikte?}

Das Fachwissen des Arztes stellt seine Hauptstärke dar und ist die Grundbedingung für das erfolgreiche Bestehen seiner Praxis. Aus der Sicht des Patienten ist die Fachkompetenz des Arztes entscheidend für die Entstehung der Vertrauensbeziehung zwischen Patient und Arzt. Die Pflege und Erweiterung dieses Fachwissens beansprucht einen wesentlichen Teil der Frei-, Arbeits- und Lebenszeit jedes Arztes.

Weil die Patienten die Fachkompetenz kaum beurteilen können, hängt der Erfolg einer Praxis auch stark von der Sozialkompetenz, der Empathie und den Kommunikationsfähigkeiten des Arztes ab.

Parallel dazu sorgt der Arzt für eine Unternehmensstrategie und Unternehmensziele, für eine sinnvolle Organisation und Einrichtung der Praxis, für das Personalmanagement wie die Personalsuche, die Auswahl, die Einarbeitung, das Delegieren von Aufgaben und die Leistungsbeurteilung. Er organisiert das Rechnungswesen und stellt die Liquidität sicher. Er stellt sich Fragen im Bereich Marketing: Wer sind die Patienten? Wie kommunizieren wir mit Patienten? Welches sind die Bedürfnisse der Patienten? Wie ist die Wahrnehmung der Praxis durch die Patienten?

Die Arztpraxis ist auch den vielfältigen Wechselbeziehungen zur Umwelt ausgesetzt. Dazu zählt das Einhalten der gesetzlichen und der brachenspezifischen Bestimmungen, die Pflege der Beziehungen zu Krankenkassen, Pharmaunternehmen und Branchenorganisationen, die Berücksichtigung der gesellschaftlichen Entwicklungen und Trends, deren erste Anzeichen in der Presse oder in Äusserungen von Patienten-

\section{Le médecin en tant \\ qu'entrepreneur et manager}

Du point de vue de la doctrine gestionnaire, chaque cabinet médical est à mettre sur un pied d'égalité avec les quelque $\mathbf{2 6 0 0 0 0}$ petites entreprises existant en Suisse. A l'instar de chaque entreprise, on trouve au cabinet médical les rôles de spécialiste, manager et entrepreneur. Alors que ces rôles sont répartis sur plusieurs personnes dans les grandes entreprises, ces tâches sont assumées le plus souvent par une seule et même personne dans les PME. Il en va de même au cabinet médical. En consacrant beaucoup de temps à ses patients, le médecin travaille essentiellement au cabinet médical et trop peu pour le cabinet médical. Des systèmes $d^{\prime}$ 'enseignement et $d^{\prime}$ 'information sont maintenant à disposition ou en cours d'élaboration pour pallier à ce déséquilibre. II est recommandé aux utilisateurs de veiller à ce qu'un tel système tienne également compte des besoins particuliers de leur domaine spécifique.

und Konsumentenschutzorganisationen wahrzunehmen sind.

\section{Wieviel Zeit bleibt für das Management?}

Wenn man der Behauptung Glauben schenkt, dass der Vorgesetzte alles delegieren kann, nur nicht die Führungsaufgaben, dann wird klar, dass der Arzt sich selbst dieser Aufgaben annehmen und dafür Zeit aufwenden muss. Konzeptionelle Aufgaben wie das Erstellen von Führungsinstrumenten kann auf die Zeit ausserhalb der Praxis verlegt werden. Aber die Mitarbeiter- oder Vorstellungsgespräche finden während der Arbeitszeit statt. In der Agenda sind also die notwendigen Zeiten dafür zu reservieren.

Unternehmensleiter von kleinen Betrieben in der Wirtschaft haben die gleichen Probleme. Die Führungstätigkeit wird zugunsten von Fachaufgaben zurückgestellt, oftmals auf ewige Zeiten. Das Tagesgeschäft beherrscht die Szene. Spontan 


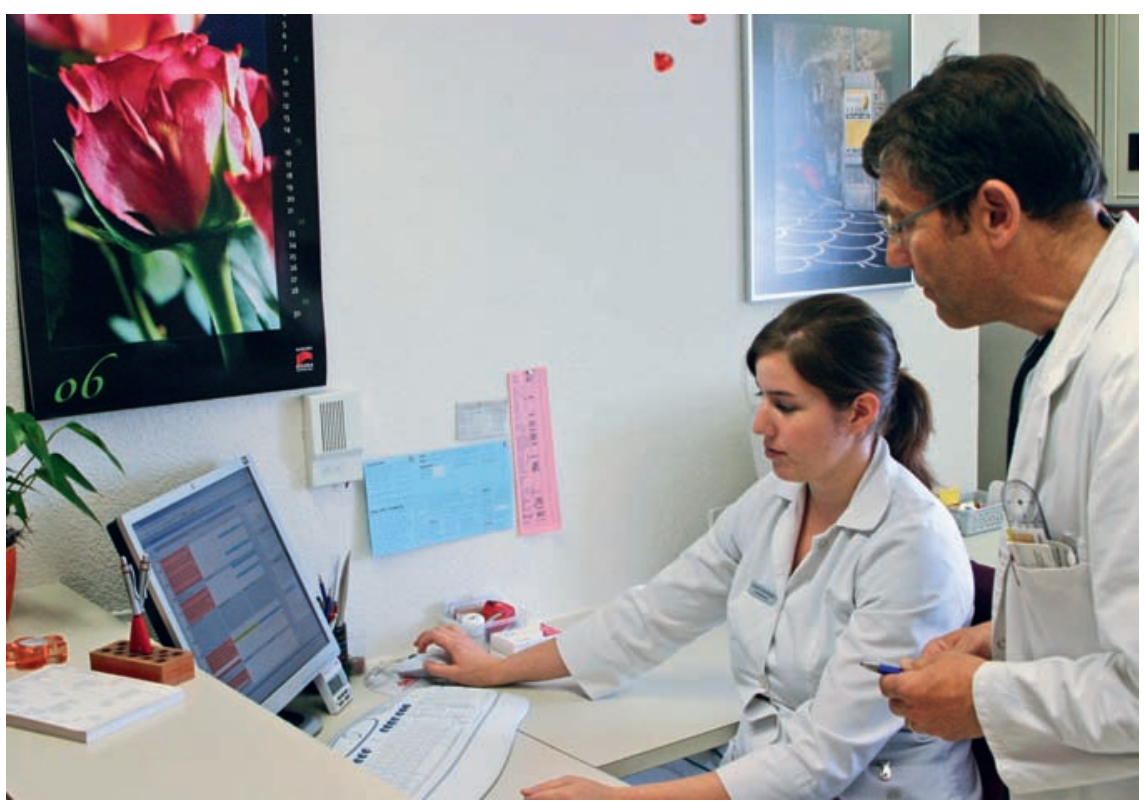

Der Arzt als Kleinunternehmer mit verschiedenen Rollen: Fachkraft, Manager und Unternehmer.

sind keine Nachteile ersichtlich, was dazu ermutigt, weiter auf die Führungstätigkeit zu verzichten. Mittel- und langfristig werden aber Probleme auftauchen, die dann auch nicht so schnell zu lösen sind.

\section{Beispiel 1}

Der Unternehmer Hans Muster betreibt mit seinen drei Angestellten eine kleine Druckerei am Stadtrand. Er ist Drucker mit Leib und Seele und steht selber jeden Tag acht Stunden an der Maschine. Er verhandelt mit Kunden, kauft Material ein, besucht die Maschinenmesse in Deutschland und zahlt gute Löhne. Er ist ein echter Profi und erteilt seinen Mitarbeitenden laufend Weisungen, was sie zu tun haben. Er hat die Druckaufträge im Griff und kennt jedes Detail. Leider hat er die Mitarbeiterführung vernachlässigt. Seine Leute sind unselbständig und entwickeln sich nicht weiter. Ohne den Chef läuft gar nichts, weil er es unterlassen hat, Aufgaben, Kompetenzen und Verantwortung zu delegieren und konsequent eine Leistungsverbesserung der Mitarbeitenden anzustreben.

\section{Beispiel 2}

Der Velohändler Fritz ist ein ausgezeichneter Mechaniker, und er versteht es auch, seine Kunden gut zu beraten. In seinem Sortiment führt er vor allem traditionelle Strassenräder und Regenbekleidung. Mit der Zeit stellt er fest, dass der Umsatz zurückgeht. Er verkauft viel weniger Velos als früher, und auch der Zubehörladen läuft nicht mehr so gut. Von einem Kollegen erfährt er, dass heute vor allem Sportbikes und Sportausrüstungen gefragt sind. Nur diese hat er nicht in seinem Sortiment, und es hat ihn auch niemand danach gefragt. Erstmals stellt er sich nun Fragen, die etwas mit Marketing zu tun haben, und hofft, dass es nicht zu spät ist.

\section{Beispiel 3}

Die Praxis von Dr. Beispiel läuft sehr gut. Neue Patienten kann er vorläufig nicht aufnehmen, weil er überlastet ist. Dann entschliesst er sich, eine junge Ärztin anzustellen, die ihn unterstützt und selbständig Patienten behandelt. Auch nach einigen Monaten ist sie aber nicht ausgelastet, und die Anzahl Patienten hat sich in dieser Praxis nicht vermehrt. Dafür gibt es Probleme in Zuständigkeitsfragen und in der Kommunikation mit den Praxisassistentinnen. Jetzt ist Dr. Beispiel gefordert als Manager. Zu seinen Pendenzen gehört die Kommunikation mit Patienten, die immer noch die Botschaft verbreiten, dass bei Dr. Beispiel keine neuen Patienten aufgenommen werden. Und praxisintern muss er die Zuständigkeiten und Kommunikationswege regeln, weil sich die Struktur der Praxis verändert hat.

Auch eine sofortige Reaktion auf der Führungsebene vermag diese Probleme nicht innert Tagen zu lösen. Aus diesem Grund sollte die Führung einer Unternehmung mit dem notwendigen Zeiteinsatz und «vorausschauend» erfolgen.

\section{Wie kommt der Arzt zu den notwendigen Managementfähigkeiten?}

Im Bereich Management existieren unzählige Angebote, den Stoff in Seminaren zu lernen. Das

\section{Innovatives Lernsystem}

Drei erfahrene Unternehmensberater - darunter der Autor - haben kürzlich das Onlineinformations- und Lernsystem «managementfürdiepraxis.ch», das auf die Bedürfnisse einer Arztpraxis ausgerichtet ist, gestartet.

In Form von kurzen, didaktisch vielfältig gestalteten Lernprogrammen stehen die wesentlichen Management- und Führungsthemen zu Verfügung. E-Coaching, moderierte Diskussionsforen und ein Downloadbereich ergänzen das Angebot.

Eine Anrechnung für diese Fortbildung in Form von Credits wurde bei der FMH beantragt.

Weitere Informationen finden Sie auf www.managementfürdiepraxis.ch. 
gibt auch die Möglichkeit - wenn es überhaupt erwünscht ist -, mit Kollegen aus anderen Fachgebieten einen intensiven Erfahrungsaustausch zu pflegen. Tatsache ist, dass es jedenfalls einige Tage braucht, um die wichtigsten Managementthemen abzudecken. Meistens sind in diesen Kursen mehrere Themen im Programm, auch solche, die im Moment vielleicht gar nicht interessieren. Der Besuch solcher Kurse bedeutet für den Arzt, dass er für diese Zeit seine Praxis schliessen muss und eine Umsatzeinbusse erleidet. Weil erfahrungsgemäss die Freizeit zu einem schönen Teil bereits für die Weiterbildung verwendet wird, steht diese Zeit nicht mehr zur Verfügung.

Eine Alternative sind Onlineangebote, die ein flexibles Lernsystem anbieten. Dabei ist wichtig, dass auch eine kompetente Begleitung in der Form von E-Coaching vorgesehen ist, weil dies markant zur Steigerung des Lerntransfers und zur Erarbeitung von massgeschneiderten Lösungen beitragen kann. Weitere Vorteile dieser Lernform sind:

- frei wählbare Lernzeit;

- ortsunabhängige Lernumgebung;

- individuelle Themenwahl nach dem Baukastenprinzip;

- individuelles Lerntempo;

- didaktisch vielfältige, interaktive Gestaltung (Text, Filme, Tests, Aufgaben, Links usw.);

- Diskussionsmöglichkeit in moderierten Foren;

- Diskretionsgrad selbst bestimmbar;

- Aktualität gewährleistet durch Newsletter;

- freie Nutzung eines Downloadbereichs mit Musterlösungen.

Solche Lern- und Informationssysteme stehen bereits zur Verfügung oder sind im Aufbau. Für den Nutzer ist es empfehlenswert, darauf $\mathrm{zu}$ achten, dass ein Lernsystem auch auf die besonderen Bedürfnisse der Branche Rücksicht nimmt. 